\title{
A propósito del artículo Impacto del aislamiento preventivo obligatorio en la actividad física diaria y en el peso de los niños durante la pandemia por SARS-CoV-2
}

\author{
About the article Impact of mandatory preventive isolation on daily physical activity and \\ weight of children during the SARS-CoV-2 pandemic
}

\author{
Karina M. Miranda-Valencia* y Carlos Egoavil-Fuentes \\ Escuela Profesional de Medicina Humana, Universidad Privada San Juan Bautista, Lima, Perú
}

Estimado señor editor:

Luego de haber leído el artículo Impacto del aislamiento preventivo obligatorio en la actividad física diaria y en el peso de los niños durante la pandemia por SARS-CoV-2, de los doctores Harold Arévalo, Miguel Urina Triana y Juan Carlos Santacruz, publicado en el número 6 del volumen 27 de su revista ${ }^{1}$, consideramos que se trata de un estudio de amplia relevancia en el campo de la cardiología por su impacto hacia el futuro en la salud de los niños. Quisiéramos acotar la importancia de reportar también la evaluación nutricional como una variable de suma importancia para determinar la presencia de sobrepeso y obesidad en niños de edad escolar en futuros estudios.

La Organización Mundial de la Salud ha declarado a la obesidad como la epidemia del siglo XXI, condición cuya relevancia no solo se centra en el incremento constante de su incidencia, sino que trasciende hacia el aumento de la morbilidad asociada (como diabetes mellitus tipo 2, dislipidemias, hipertensión arterial y ciertos tipos de cáncer) que compromete muy seriamente la salud a temprana edad. En los últimos años, varios estudios de confiabilidad mencionan a la obesidad infantil como un potencial factor de riesgo a futuro ${ }^{2}$.

Por otra parte, en el estudio no se tomó en cuenta si los niños habían adquirido un hábito alimenticio inadecuado, y el consumo de azúcar diario es de gran relevancia, pues el consumo excesivo de dichos insumos puede influir en un aumento excesivo de peso en los niños.

El consumo de azúcar en sus diferentes presentaciones, tales como son los refrescos o bebidas azucaradas, incrementa el riesgo de obesidad y los riesgos cardiovasculares en el futuro. El primer factor de incremento calórico lo proporcionan los refrescos o bebidas azucaradas, el cual es de $150 \mathrm{kcal}$ por cada $350 \mathrm{ml}$ aproximadamente. Hubiera sido interesante conocer si estos niños consumían estas bebidas y la frecuencia con que lo hacían.

Las bebidas azucaradas en los niños y adolescentes presentan diversos efectos negativos, tales como aumento de la adiposidad, aumento de la presión arterial, resistencia a la insulina, diabetes tipo 2, síndrome metabólico, problemas dentales y problemas comportamentales ${ }^{3}$.

Es importante mencionar también que el consumo de frutas y verduras es de vital importancia dentro de la dieta de un niño. Hubiera sido interesante conocer
Correspondencia:

*Karina M. Miranda-Valencia

E-mail: karina.miranda@upsjb.edu.pe licencia CC BY-NC-ND (http://creativecommons.org/licenses/by-nc-nd/4.0/).

Fecha de recepción: 10-06-2021

Fecha de aceptación: 30-11-2021

DOI: 10.24875/RCCAR.21000076
Disponible en internet: 22-02-2022

Rev Colomb Cardiol. 2022;29(1):117-118

www.rccardiologia.com 
con qué frecuencia consumían frutas y verduras, sobre todo por el incremento de familias que durante la pandemia crearon en casa un ambiente obesogénico.

Según el Dr. José L. Medina-Valdivia: «En el Perú, en consultorio externo de pediatría del Hospital Regional Moquegua, de un total de 170 niñas y niños de 5 a 10 años atendidos se encontró 88 niños (51.8\%) con sobrepeso y obesidad, 36 niñas y niños (21.2\%) con sobrepeso, y 52 niñas y niños (30.6\%) con obesidad» ${ }^{4}$.

Una evaluación sobre la obesidad en los niños es importante. En un estudio realizado en Verona, Italia, en 2020, se constató el aumento de peso en los niños tras unos malos hábitos alimenticios, los cuales constaban en el excesivo consumo de carne roja, pasta y meriendas. En dicho estudio se confirmó el cambio de hábitos saludables que tenían los niños, lo que indica que a los niños con obesidad les va peor en los programas de estilo de vida de control de peso mientras están en casa, a diferencia de cuando participan en su plan de estudios escolar ${ }^{5}$.

La obesidad presenta múltiples causas de inicio, en el que se encuentran comprometidos los factores genéticos, los hábitos de buena alimentación y las características del ambiente. De igual manera se presentan determinantes implícitos, tales como la universalización, el entorno económico, la capacitación de la población y el desarrollo urbano, los cuales son factores que favorecen el cambio en los estilos de vida saludable en los diversos grupos humanos ${ }^{6,7}$.

Con todo lo expuesto, esta carta pretende incentivar la investigación en las características destacadas previamente, pues en nuestro país tampoco existen muchos estudios al respecto. Además, dichas investigaciones podrán servir para identificar casos de sobrepeso y obesidad a temprana edad y poder actuar a tiempo, así como conocer nuevas medidas que ayuden a disminuir la prevalencia de obesidad infantil en nuestro medio.

\section{Financiamiento}

Los autores declaran que la redacción de esta carta no tuvo financiamiento.

\section{Conflicto de intereses}

Los autores declaran no tener ningún conflicto de intereses.

\section{Responsabilidades éticas}

Protección de personas y animales. Los autores declaran que para esta investigación no se han realizado experimentos en seres humanos ni en animales.

Confidencialidad de los datos. Los autores declaran que en este artículo no aparecen datos de pacientes.

Derecho a la privacidad y consentimiento informado. Los autores declaran que en este artículo no aparecen datos de pacientes.

\section{Bibliografía}

1. Arévalo H, Urina Triana M, Santacruz J. Impacto del aislamiento preventivo obligatorio en la actividad física diaria y en el peso de los niños durante la pandemia por SARS-CoV-2. Rev Col Cardiol. 2020;27:575-82.

2. Organización Mundial de la Salud. Obesidad y sobrepeso. OMS; 2021. (Consultado 06-05-2021.) Disponible en: https://www.who.int/es/newsroom/fact-sheets/detail/obesity-and-overweight

3. Ruiz Castellanos E. Relación entre el consumo de bebidas azucaradas e indicadores de adiposidad en niños y adolescentes de Bogotá: estudio FUPRECOL 2014. Magister, Universidad Colegio Mayor de Nuestra Señora del Rosario; 2016. Disponible en: https://repository.urosario.edu.co/ bitstream/handle/10336/13125/Tesis\%20Bebidas\%20Azucaradas $\% 20$ ErikaRuiz\%20Urosario\%20dic\%202016.pdf?sequence=1

4. Medina-Valdivia JL. Sobrepeso y obesidad infantil en el Hospital Regional Moquegua. Rev Fac Med Hum. 2019;19:16-26.

5. Pietrobelli A, Pecoraro L, Ferruzzi A, Heo M, Faith M, Zoller T, et al. Effects of COVID-19 lockdown on lifestyle behaviors in children with obesity living in Verona, Italy: a longitudinal study. Obesity. 2020;28:1382-5

6. Vicente-Sánchez B, García K, González-Hermida A, Saura-Naranjo C. Sobrepeso y obesidad en niños de 5 a 12 años. Revista Finlay [revista en Internet]. 2017 [citado 2022 Ene 21]; 7(1):[aprox. 6 p.]. Disponible en: http://www.revfinlay.sld.cu/index.php/finlay/article/view/445

7. Dávila-Torres J, González-Izquierdo JJ, Barrera-Cruz A. Panorama de la obesidad en México. Rev Med Inst Mex Seguro Soc. 2015;53:241-9. 